\title{
BMJ Open Use of touch screen tablets to support social connections and reduce responsive behaviours among people with dementia in care settings: a scoping review protocol
}

\author{
Lillian Hung (D , , Annette Berndt, ${ }^{2}$ Christine Wallsworth, ${ }^{2}$ Neil Horne, ${ }^{2}$ \\ Mario Gregorio, ${ }^{2} \mathrm{Jim}$ Mann, ${ }^{2}$ Cathy Son, ${ }^{3}$ Ainsley Camps, ${ }^{4}$ Bryan Chow, ${ }^{5}$ \\ Ryan O'Neill, ${ }^{5}$ Habib Chaudhury ${ }^{6}$
}

To cite: Hung L, Berndt A, Wallsworth C, et al. Use of touch screen tablets to support social connections and reduce responsive behaviours among people with dementia in care settings: a scoping review protocol. BMJ Open 2019;9:e031653. doi:10.1136/ bmjopen-2019-031653

- Prepublication history and additional material for this paper are available online. To view these files, please visit the journal online (http://dx.doi org/10.1136/bmjopen-2019031653).

Received 14 May 2019

Revised 04 November 2019 Accepted 05 November 2019

Check for updates

(C) Author(s) (or their employer(s)) 2019. Re-use permitted under CC BY-NC. No commercial re-use. See rights and permissions. Published by BMJ.

For numbered affiliations see end of article.

Correspondence to

Dr Lillian Hung;

lillian.hung@vch.ca

\section{ABSTRACT}

Introduction The disabilities associated with dementia make the adjustment to staying in a care setting stressful. Separation from family can exacerbate the effects of stress. The use of touch screen tablets such as an iPad may offer potential to support the person with dementia staying in a care setting. Although electronic devices are used among people with dementia for a variety of purposes, a comprehensive review of studies focusing on their impact in care settings for social connection and patient/resident behaviour is lacking. This scoping review will focus on the use of touch screen tablets to support social connections and reducing responsive behaviours of people with dementia while in a care setting, such as a hospital ward. Methods and analysis This scoping review will follow Joanna Briggs Institute scoping review methodology. The review team consists of two patient partners and three family partners, a nurse researcher, a research assistant and an academic professor. All authors including patient and family partners were involved in preparing this scoping review protocol. In the scoping review, we will search the following databases: MEDLINE, AgeLine, Cochrane, CINAHL, PsycINF0 and IEEE. Google and Google Scholar will be used to search for additional literature. A hand search will be conducted using the reference lists of included studies to identify additional relevant articles. Included studies must report on the impact of using a touch screen technology intervention that involves older adults with dementia in care settings, published in English since 2009.

Ethics and dissemination This review study does not require ethics approval. By examining the current state of using touch screen tablets to support older people with dementia in care settings, this scoping review can offer useful insight into users' needs (eg, patients' and care providers' needs) and inform future research and practice. We will share the scoping review results through conference presentations and an open access publication in a peer-reviewed journal.

\section{INTRODUCTION}

The number of people worldwide being diagnosed with dementia is rising sharply.
Strengths and limitations of this study

- This review involves knowledge users, including front-line clinicians, patient and family partners in setting the research questions and in designing and writing of the review protocol.

- This is a novel and timely review to understand how touch screen tablets may support social connections and reduce responsive behaviours among people with dementia in care settings.

- Although the results may have useful value to health professionals by providing a comprehensive evidence base to help with their planning and decisionmaking regarding the intervention, implications for practice are limited by the fact that an assessment of methodological quality of the included studies will not be performed.

- The review is limited to literature published in English; publications in other language will not be considered.

- Useful strategies for touch screen applications in home settings will not be captured.

According to WHO, every $3 \mathrm{~s}$ someone is diagnosed with dementia. An estimated 50 million people worldwide are currently living with dementia, and this number will triple by 2050. In Canada, where this review was done, the Alzheimer Society of Canada reports that half a million people are currently living with dementia, with 25000 new cases being diagnosed every year. ${ }^{2}$ The signs and symptoms linked to dementia can be understood in three stages: (1) early stage with common symptoms such as forgetfulness and becoming lost in familiar places, (2) middle stage with symptoms like experiencing behavioural changes, including wandering and repeated questioning and (3) late stage with symptoms that include an increased need for assisted 
self-care. ${ }^{1}$ With no cure in the foreseeable future, the focus is on care to maintain the quality of life of people affected by the disease. People with dementia also face challenges with their ability to communicate in-depth ideas and express emotions verbally. ${ }^{3}$ The behavioural and psychological symptoms of dementia (commonly referred to as BPSD), which include agitation, anxiety, depression and hallucinations, can disrupt family and care relationships of people with dementia. ${ }^{4}$ People with dementia may also use responsive behaviour (eg, calling out, striking) to express unmet needs. ${ }^{5}$

Adjustment to staying in a care setting is stressful, and separation from family and friends can intensify that stress. ${ }^{6}$ Opportunities for communication and connection with family and friends may be reduced by work pressures as well as travel time to the care setting. These issues have a negative effect on the number and quality of family visits. Family relationships experience stress related to a feeling of having less input or control over how care is planned and carried out, creating a disconnect for families trying to adjust to their new role in care. ${ }^{3}$ Activities and technology that support and maintain communication between people with dementia and their families and care providers may contribute to maintaining quality of life and providing person-centred care. ${ }^{7}$ For example, a recent study ${ }^{8}$ investigated the use of Skype on iPads with older residents in a long-term care setting; residents reported positively about being able to talk with family and explained how their declined health condition made it difficult for them to leave the care setting to visit their family at home in the community. Loss of social connection is linked to a more rapid cognitive decline; whereas activities that facilitate a positive connection with others is intrinsic to maintaining mental health. ${ }^{4}$ Technology designed to assist in maintaining and supporting the connection with family and formal care providers has been studied to identify its value and potential to support care for people with dementia. ${ }^{369}$ Yet, the evidence on the feasibility and impact of the use of touch screen tablets for people with dementia in care settings has not been well established. There is a need to identify practical strategies to understand what makes a technological intervention work for the specific population of people with dementia and why; we also need to learn about the problems and barriers that should be addressed in care settings. ${ }^{10}$ For example, the study conducted by Hung et at highlights the complexity of knowledge and skills required among healthcare staff to enable them to play family videos on a touch screen tablet (iPad) to support people with early to late stage of dementia care in a hospital ward.

Touch screen tablets have been studied for their accessibility for use by people with dementia. A literature review by Joddrell and Astell ${ }^{11}$ notes that several physical characteristics of touch screen tablets (such as iPad and Android) make them easier to use by people with dementia. Doing away with external keyboards and the mouse reduces the amount of cognitive load required to use touch screen tablets. Also, the touch screen interface makes the tablet easy to use. ${ }^{12}$ In addition, most touch screen tablets are small enough to be carried around and are easily customised to hold materials and information that can be adapted and personalised for a specific individual or group of individuals. ${ }^{13}$ A recent study showed positive results of using videoconferencing on iPads to support family connection, but staff and residents also reported issues related to physical frailty and cognitive impairment. ${ }^{8}$ In an earlier study, a telepresence robot with a video conferencing function, Giraff, was tested among residents with dementia in a long-term care facility. ${ }^{3}$ However, poor internet connectivity limited the extent to which family members could use the telepresence robot in the care setting. Concerns have been raised that people with dementia may not accept new touch screen tablets or may not know how to use them due to their newness and unfamiliarity. ${ }^{14}$ More knowledge is needed to understand how touch screen tablets have been used in the care settings and what support successful application.

A preliminary search for existing literature reviews was conducted on 15 December 2018 in the Joanna Briggs Institute (JBI) Database of Systematic Reviews and Implementation Reports, Cochrane Database of Systematic Reviews, TRIP database and PROSPERO. No systematic reviews on the topic of using touch screen tablets to support people with dementia in care settings were identified. In the literature, a scoping review on the topic of using touch screen tablets ${ }^{15}$ by people with dementia has been conducted in the context of peoples' homes, not formal care settings.

\section{STUDY OBJECTIVE}

The objective of this scoping review is to summarise and synthesise existing knowledge on the use of touchscreen tablets to support the connection of people diagnosed with dementia with their families and care providers and reduce the responsive behaviours of people with dementia in care settings, such as care facilities or hospitals.

In this scoping study, we want to know: what are the effective ways of using a touch screen tablet to support a connection between care providers and people with dementia in hospitals? And, what strategies enable family members and care teams to use touch screen tablets effectively to make people living with dementia more comfortable in hospital wards? Data extracted from each study will be mapped and presented in a form that reflects the objective of this scoping review. The mapping of available evidence will provide directions for future research and inform our next study, involving the use of a touch screen tablet (iPad) loaded with videos prerecorded by family members to reduce responsive behaviours such as hitting out during care, and thereby improving the quality of life for people with dementia. In our next study, we will use the evidence mapped in the scoping review to engage hospital staff and families to coproduce practical strategies for supporting the use of touch screen tablets (iPads) to innovate and improve dementia care in hospital wards. 


\begin{tabular}{|c|c|}
\hline PCC & Inclusion criteria \\
\hline Population & $\begin{array}{l}\text { Older adults (aged } 60 \text { or older) with all types } \\
\text { and all stages of dementia. }\end{array}$ \\
\hline Concept & $\begin{array}{l}\text { Intervention (touch screen tablets). } \\
\text { Outcomes (social connection, responsive } \\
\text { behaviours). }\end{array}$ \\
\hline Context & $\begin{array}{l}\text { Care settings (hospitals, day care, long-term } \\
\text { care homes). }\end{array}$ \\
\hline
\end{tabular}

PCC, Population, Concept, and Context.

\section{METHODS}

To meet the objective stated above, we will conduct a methodologically rigorous scoping review by following the methodology for scoping reviews developed by JBI. ${ }^{16}$ A scoping review is appropriate for this study because it provides an overview of the relevant literature in a field that is underdeveloped in order to identify the key themes and contexts within a research topic. ${ }^{16}{ }^{17}$ Unlike systematic reviews which target highly focused questions such as the effectiveness of a particular intervention based on a precise set of outcomes, scoping reviews are used to determine the extent of existing evidence, to summarise existing evidence for dissemination and to identify avenues for future research. ${ }^{18} \mathrm{An}$ assessment of methodological quality will not be performed in this review because the goal of scoping reviews is not to produce a critical appraisal. ${ }^{19}{ }^{20}$ In line with the JBI guideline, this scoping review is intended to determine what range of evidence is available on a topic regardless of quality. ${ }^{21}$ In addition to summarising research findings, scoping reviews may be undertaken to identify research gaps for the future research. ${ }^{19}$ If implications for practice are produced from the scoping review, the JBI Grades of Recommendation must be used. ${ }^{22}$

This protocol provides an important plan in order to conduct the review and also allows transparency of process. ${ }^{16}$ JBI uses the PCC (Population, Concept, and Context) mnemonic to guide the formulation of scoping review questions and inclusion criteria. See table 1 for the PCC and inclusion criteria. In terms of population, we will consider studies that include older people diagnosed with all types and all stages of dementia. We have limited the review by participants aged 60 or older because older adults with dementia are at risk of lacking social connection. ${ }^{823}$ For concept, we will include studies that provide information about any touch screen tablet intervention and outcomes on social connection and responsive behaviours. Regarding context, we will consider studies that examine the use of touch screen tablets in formal care settings such as hospitals, day care and long-term care homes.

To address the PCC and the objective of the review, we formulated two scoping review questions:

1. What has been reported in the literature regarding the impact of touchscreen tablets in supporting social connections and reducing responsive behaviours of people with dementia in care settings?

2. What strategies may support family members and care teams in the use of touch screen tablets with people with dementia?

\section{The study team}

The scoping review will be conducted by a diverse team including two patient partners, three family partners, two physicians, two university students and a nurse researcher. One academic professor will serve as a mentor to provide guidance and support for the study. The review will be performed through five stages: conducting broad searches, refining selection criteria, study selecting and reviewing results, mapping literature and summarising results. The Preferred Reporting Items for Systematic Reviews and Meta-Analyses for Scoping Reviews (PRISMA-ScR) checklist, which is consistent with the JBI methodology for scoping review will also be used to structure the reporting of the scoping review. ${ }^{24}$ The checklist is available as an online supplementary file 1 .

\section{Conducting broad searches}

A search approach which is comprised of three steps will be performed as recommended by the JBI. ${ }^{16}$ See online supplementary file 2 for the search process and search terms. Step 1 involves identifying the terms for the search through an analysis of the words contained in the title and abstract of retrieved articles, and of the index terms which are used to describe the articles. We have consulted with a gerontology research librarian in the university to select the initial search terms. We applied the initial search terms to conduct a search in two databases: MEDLINE \& AgeLine. Search terms included touch screen, iPad, tablet, mobile technology, assistive technology, dementia, Alzheimer. See details of Boolean operators and truncation in online supplementary file 2 . This initial search is then followed by an analysis of the text words contained in the title and abstract of retrieved articles, and of the index terms used to describe the articles. The objective was to identify the terms that make it possible to find the relevant articles. ${ }^{22}$ Studies published in English from 2009 to the present will be included. We framed our search parameters to span 10 years because our preliminary search identified limited studies before 2009. After the launch of Apple iPad in 2010, there was an increase in interest and studies about using touch screen tablets among older people, including people with dementia. ${ }^{25}$ The search will also be extended to Google for grey literature (ie, organisational reports, newsletters and other articles not indexed in library database in Google Scholar). For the Google search, we will perform phrase searching. See online supplementary file 2 for the details of phrase searching. All empirical peer-reviewed publications as well as documents from the grey literature that examine the use of touch screen tablets for older adults with dementia in care settings will be considered for inclusion. Studies in all designs, including quantitative and 
qualitative research, mixed methods, systematic reviews as well as small feasibility pilots and user experience report will be included.

\section{Refining selection criteria}

In step 2, we will search identified keywords and index terms across all selected databases: MEDLINE, AgeLine, Cumulative Index to Nursing and Allied Health, Cochrane, PsycINFO and IEEE. Inclusion and exclusion criteria will be applied to select articles. Articles will be included if they: (1) focused on older people with all types and all stages of dementia, (2) targeted any intervention involving touch screen tablets, (3) were studied in formal care settings (eg, nursing homes, day programme and hospitals) and (4) describe outcomes that had relevance in promoting social connection and improving well-being (eg, reduction of responsive behaviours, improvement in well-being and quality of life). See online supplementary file 3 for the data extraction instrument. ${ }^{22}$ The data extracted will include specific details about the population, concept, context, study methods and key findings relevant to the review objective.

In step 3, reference lists of articles that fit our search criteria will then be hand searched for additional sources. Google Scholar will be used to find published articles, organisational reports and related articles. All reports on the same study will be considered if the relevant outcomes of interest are different; otherwise, only the most recent report will be included in the review.

\section{Study selection and reviewing results}

A bibliographic reference management tool, Mendeley, will be used to ensure that all references and articles are systematically organised. All identified relevant articles will be uploaded into Mendeley; duplicates will be removed. The review process will involve two levels of screening: a title and abstract review followed by a full-text review. In the first level of screening, three investigators will independently screen the title and abstract for relevancy. In the second level of screening, the full text of studies that may meet the inclusion criteria will be retrieved and read independently by the three investigators to confirm inclusion. Studies that do not meet the inclusion criteria will be excluded and the reasons for exclusion will be presented in a PRISMA flow diagram. ${ }^{22}$ Any disagreements that arise between the reviewers will be resolved through discussion. If needed, the first author may also take specific issues to the whole research team and facilitate discussion until agreement is reached. A data analysis software programme, NVivo V.12, will be used to identify and extract data for full-text review in selected articles. The data extracted will include specific details about the population, concept, context, study methods and key findings relevant to the review questions. The data extraction tool will be pilot tested having three investigators to complete extraction from three data sources and comparing results. The draft data extraction tool will be modified and revised as necessary during the process of extracting data from each included study. Modifications will be detailed in the full scoping review report.

\section{Mapping literature}

The process of charting the results provides the reader with a logical and descriptive summary of the results that aligns with the objective and questions of the scoping review. ${ }^{21}$ Relevant descriptive information and data will be extracted and charted from studies included in the scoping review using a data extraction instrument (online supplementary file 3) derived from the objectives of the research. The template of the data extraction instrument may be expanded and adapted during the course of the review, and changes reported in the published scoping review. ${ }^{22}$ We will map the selected papers by domains: author and country, setting, participants, research design, measures, strategies and impact/outcomes. A summary table of included studies will be developed. In research meetings, the whole team including patient and family partners will take part in analysing the extracted data sorted according to potential themes. We will compare and discuss different interpretations to resolve conflicts. The extracted data/charted results will be collectively evaluated and collated into categories of key findings for the final report presentation

\section{Summarising results}

We will report the findings of the review in a manuscript of a peer-reviewed open access journal. A PRISMA flow diagram will be included to describe the review process. Also, we will provide a literature table to give a summary of characteristics of the selected papers, including study location, year of publication, study population and sample size, methods, intervention type, impacts, barriers to technology use and strategies to overcome barriers. A narrative summary will accompany the charted results and describe how the results relate to the review objective and questions.

\section{Patient and public involvement}

Patient and family partners were recruited from a local community organisation, the Community Engagement Advisory Network (CEAN), which provides training and support in a variety of ways, including education workshops, public forums, as well as online and brochure materials for patient and family as partners. More information about how CEAN supports patient and public involvement can be found in http://cean.vch.ca. Two patient and three family partners (coauthors of the protocol) were involved in preparing the scoping review protocol that underpinned the priority focus, and research directions. Through regular research meetings and conversations with patient and family partners, we collectively discussed and jointly decided research questions, what to include and exclude in the scoping review. Actively involving research knowledge users (patients, families and clinicians) in the project challenges the current paradigm of a historical, paternalistic and imbalanced relationship between 
academic researchers and knowledge users. Patient and family partners were involved in the design of the scoping review protocol by openly sharing their priorities and making decision together with the team about the study plan. As a team, we discussed and formally agreed on the time required to participate in the research. One family partner participated in writing this protocol. Patient and family partners will continue to work with researchers and clinicians to complete the scoping review, contributing to team discussion and providing feedback on the results charted. Patient and family partners are committed to writing parts of the scoping review report for publication and disseminating results in conference presentations. In the scoping study, we will apply Guidance for Reporting Involvement of Patients and the Public ${ }^{26}$ in evaluating the involvement of patient and family as partners in research.

\section{Ethics and dissemination}

Since the methodology of the study consists of collecting data from publicly available articles, it does not require ethics approval. As a team that includes academics and clinicians working with people living with dementia and family of person with dementia, we engage in team reflection in regular meetings and use the guidance of the ethical framework 'ASK ME' specifically developed for coresearch with people with dementia. ${ }^{27}$ ASK ME stands for Avoid assumption, Support the person to do their best, consider Knowledge needed to be put into action, Meet early and regularly and consider Ethical sensitivity and responsibility. Our process is also informed by the lessons learnt in a previous patient co-research study. ${ }^{28}$

This scoping review protocol presents a transparent and comprehensive methodology. The completed scoping review will be submitted for publication in an open-access and peer-reviewed interdisciplinary journal, and the results will be presented at relevant conferences. The findings will also be used to guide our next research project, titled 'Innovating Together: Working with technologies in dementia care'.

\section{DISCUSSION}

The results of the scoping study will provide a summary of evidence about the impact of touch screen tablets in supporting social connections and reducing responsive behaviour of people with dementia in care settings. In the literature, a scoping review ${ }^{15}$ on the topic of using touch screen tablets by people with dementia has been conducted in the context of peoples' homes, not formal care settings. Touch screen tablets have been recognised as being useful in building rapport, increasing engagement with and discovering interests and capabilities of people with dementia for caregiving staff and in facilitating continued connection and communication with family members. ${ }^{29}$ Research on the use of iPads in oneto-one, small-group and large-group activities in longterm care homes indicates that appropriate use of iPads promotes positive emotions and social connections among residents, staff and family care partners. ${ }^{29}{ }^{30}$ Potentially, touch screen tablets could be used as a viable psychosocial intervention to relieve emotional distress and psychological distress among people with dementia ${ }^{31} 32$ and to promote person-centred care ${ }^{33}$ in which people's desires and values are respected. Even though there are benefits to using a touch screen tablet, there is still limited evidence to aid care providers and families in the use of such technology.

This is a novel and timely review aimed at how touch screen tablets may support social connections and reduce responsive behaviours in dementia care. The data in our study may offer useful insights to inform possible strategies that support family members and care teams to use touch screen tablets with people who are living with dementia. Based on the scoping review findings, our next study will further investigate strategies to overcome barriers for families using videos via touch screen tablets (iPad) to support dementia care in the hospital setting. We will engage hospital staff and families to co-design practical approaches for the adoption of touch screen technology in hospital wards. We plan to develop a toolkit containing (1) a public information website, (2) a how to' brochure for supporting family partnership and (3) other practical tools to support the use of touch screen tablets as a psychosocial intervention in the hospital setting. We anticipate the next research step will provide useful knowledge and practical support for clinicians to integrate touchscreen technology in care practice. Co-designing is a novel approach in developing technology interventions in the field of gerontology to ensure knowledge co-production is grounded in the lived experience of its users. ${ }^{34}$

The scoping review will only include literature published in English so studies published in other languages will not be captured. Our search only includes studies in care setting; therefore, helpful and applicable strategies for touch screen applications in home settings will be missed. While there may be a possible limitation in implications for practice as methodological assessment of the included studies will not be performed, a comprehensive summary of existing evidence may be useful to inform future research and practice.

\section{Author affiliations}

${ }^{1}$ Gerontology, Simon Fraser University, Vancouver, British Columbia, Canada ${ }^{2}$ Community Engagement Advisory Network, Vancouver Coastal Health Authority, Vancouver, British Columbia, Canada

${ }^{3}$ School of Nursing, Trinity Western University, Langley, British Columbia, Canada ${ }^{4}$ Social Science, Simon Fraser University, Burnaby, British Columbia, Canada ${ }^{5}$ Medicine, The University of British Columbia, Vancouver, British Columbia, Canada ${ }^{6}$ Gerontology, Simon Fraser University, Burnaby, British Columbia, Canada

\section{Twitter Lillian Hung @nurselillian}

Acknowledgements The authors wish to thank the assistance of the Gerontology Research Librarian, Nina Smart at Simon Fraser University.

Contributors LH led the conceptualisation, design and development of this study and protocol. All authors were involved in developing the research questions and the scoping review plan. $\mathrm{LH}, \mathrm{CS}, \mathrm{AB}$ and $\mathrm{AC}$ drafted the protocol. HC provided guidance to the study conceptualisation and design and revised this manuscript 
for content and clarity. All authors actively engaged in team discussions in regular research meetings for the development of the protocol and approved the publishing of this protocol manuscript.

Funding The first author was supported by the Alzheimer Society of Canada Postdoctoral Fellowship.

Competing interests None declared.

Patient consent for publication Not required.

Provenance and peer review Not commissioned; externally peer reviewed.

Open access This is an open access article distributed in accordance with the Creative Commons Attribution Non Commercial (CC BY-NC 4.0) license, which permits others to distribute, remix, adapt, build upon this work non-commercially, and license their derivative works on different terms, provided the original work is properly cited, appropriate credit is given, any changes made indicated, and the use is non-commercial. See: http://creativecommons.org/licenses/by-nc/4.0/.

ORCID iD

Lillian Hung http://orcid.org/0000-0002-7916-2939

\section{REFERENCES}

1 World Health Organization. Dementia key facts, 2019. Available: https://www.who.int/en/news-room/fact-sheets/detail/dementia [Accessed $31 \mathrm{Mar} 2019]$.

2 Alzheimer Society of Canada. Dementia numbers in Canada., 2019. Available: https://alzheimer.ca/en/Home/About-dementia/What-isdementia/Dementia-numbers [Accessed 9 Oct 2019].

3 Moyle W, Jones C, Cooke M, et al. Connecting the person with dementia and family: a feasibility study of a telepresence robot. BMC Geriatr 2014;14:1-11.

4 Savundranayagam MY, Lee C. Roles of communication problems and communication strategies on Resident-Related role demand and role satisfaction. Am J Alzheimers Dis Other Demen 2017;32:116-22.

5 Hung L, Son C, Hung R. The experience of hospital staff in applying the gentle persuasive approaches to dementia care. J Psychiatr Ment Health Nurs 2019;26:1-10.

6 Hung L, Au-Yeung A, Helmer C, et al. Feasibility and acceptability of an iPad intervention to support dementia care in the hospital setting. Contemp Nurse 2018;54:350-61.

7 Siniscarco MT, Love-Williams C, Burnett-Wolle S. Video Conferencing: an intervention for emotional loneliness in long-term care. Act Adapt Aging 2017;41:316-29.

8 Moyle W, Jones C, Murfield J, et al. 'For me at 90, it's going to be difficult': feasibility of using iPad video-conferencing with older adults in long-term aged care. Aging Ment Health 2019;0:1-4.

9 Loi SM, Mazur A, Huppert D, et al. A pilot study using "apps" as a novel strategy for the management of challenging behaviors seen in people living in residential care. Int Psychogeriatr 2017;29:637-43.

10 Vichitvanichphong S, Talaei-Khoei A, Kerr D, et al. Assistive technologies for aged care. Inf Technol People 2018;31:405-27.

11 Joddrell P, Astell AJ. Studies involving people with dementia and Touchscreen technology: a literature review. JMIR Rehabil Assist Technol 2016;3:e10.

12 Dynes K. iPad use to enhance conversations between persons with dementia and their family caregivers, 2018. Available: https://ir.lib. uwo.ca/etd/5617

13 Joddrell P, Astell AJ. Maximising the value of TOUCHSCREEN tablet devices for people living with dementia. Alzheimer's \& Dementia 2016;12:P258-9.
14 Perry J, Beyer S. Ethical issues around telecare: the views of people with intellectual disabilities and people with dementia. J Assist Technol 2012;6:71-5.

15 Hitch D, Swan J, Pattison R, et al. Use of touchscreen tablet technology by people with dementia in homes: a scoping review. $J$ Rehabil Assist Technol Eng 2017;4.

16 Peters M, Godfrey C, Khalil H. The Joanna Briggs Institute Reviewers' Manual 2015: Methodology for JBI scoping reviews, 2015.

17 Pham MT, Rajić A, Greig JD, et al. A scoping review of scoping reviews: advancing the approach and enhancing the consistency. Res Synth Methods 2014;5:371-85.

18 Gibson G, Newton L, Pritchard G, et al. The provision of assistive technology products and services for people with dementia in the United Kingdom. Dementia 2016;15:681-701.

19 Munn Z, Peters MDJ, Stern C, et al. Systematic review or scoping review ? Guidance for authors when choosing between a systematic or scoping review approach 2018:1-7.

20 Armstrong R, Hall BJ, Doyle J, et al. 'Scoping the scope' of a cochrane review. J Public Health 2011;33:147-50.

21 Peters M, Godfrey C, Khalil H, et al. The Joanna Briggs Institute Reviewers' Manual: 2015 edition/supplement 2015.

22 Peters M, Godfrey-smith P, Mcinerney P. Guidance for the Conduct of JBI Scoping Reviews. In: Joanna Briggs Institute Reviewer's Manual. South Australia: Joanna Briggs Institute, 2019. https:// reviewersmanual.joannabriggs.org/

23 Moyle W, Arnautovska U, Ownsworth T, et al. Potential of telepresence robots to enhance social connectedness in older adults with dementia: an integrative review of feasibility. Int Psychogeriatr 2017;29:1951-64.

24 Tricco AC, Lillie E, Zarin W, et al. PRISMA extension for scoping reviews (PRISMA-ScR): checklist and explanation. Ann Intern Med 2018;169:467-73.

25 Schikhof $Y$, Goumans M, Joddrell P, et al. Comparing recent reviews about touchscreens for dementia with lessons learnt from the field. Stud Health Technol Inform 2017;242:1-4.

26 Staniszewska S, Brett J, Simera I, et al. GRIPP2 reporting checklists: tools to improve reporting of patient and public involvement in research. Res Involv Engagem 2017;3:1-11.

27 Mann J, Hung L. Co-research with people living with dementia for change. Action Res2018;147675031878700.

28 Hung L, Berndt A, Wallsworth C, et al. Involving patients and families in a social robot study. Patient Exp J 2019;6:66-74.

29 Swan J, Hitch D, Pattison R, et al. Meaningful occupation with iPads: Experiences of residents and staff in an older person's mental health setting. Br J Occup Ther 2018;81:649-56.

30 Evans SB, Bray J, Evans SC. The iPad project: introducing iPads into care homes in the UK to support digital inclusion. Gerontechnology 2017;16:91-100.

31 Vahia IV, Kamat R, Vang C, et al. Use of tablet devices in the management of agitation among inpatients with dementia: an openlabel study. Am J Geriatr Psychiatry 2017;25:860-4.

32 Upton D, Upton P, Jones T, et al. Evaluation of the impact of touch screen technology on people with dementia and their carers within care home settings, 2011. Available: https://core.ac.uk/download/ pdf/51151130.pdf

33 Hung L, Phinney A, Chaudhury $\mathrm{H}$, et al. "Little things matter!" Exploring the perspectives of patients with dementia about the hospital environment. Int J Older People Nurs 2017;12:1-11.

34 Wherton J, Sugarhood P, Procter R, et al. Co-production in practice: how people with assisted living needs can help design and evolve technologies and services. Implement Sci 2015;10:1-10. 